\title{
Effects of axial-loaded magnetic resonance imaging of lumbar spine on dural sac and lateral recesses
}

\author{
Deniz Bulja1*, Jasna Strika², Merim Jusufbegović1, Muris Bečirčić', Adnan Šehić3 ${ }^{3}$ Fuad Julardžijaª , \\ Adnan Beganović1, Fuad Zukić1, Sandra Vegar-Zubović
}

\begin{abstract}
${ }^{1}$ Radiology Clinic, Clinical Center University of Sarajevo, Sarajevo, Bosnia and Herzegovina, ${ }^{2}$ Department of Radiology, Cantonal Hospital Zenica, Zenica, Bosnia and Herzegovina, ${ }^{3}$ Department of Radiological Technology, Faculty of Health Studies, University of Sarajevo, Sarajevo, Bosnia and Herzegovina
\end{abstract}

\section{ABSTRACT}

Introduction: Axial-loaded magnetic resonance imaging (MRI), which can simulate an upright position of the patient may cause a significant reduction of the dural sac cross-sectional area (DCSA) compared with standard MRI, thus providing valuable information in the assessment of the lumbar spinal canal. The purpose of this study was to investigate excessiveness of the change in DCSA and depth of lateral recesses (DLRs) before and after axial-loaded imaging in relation to body mass index (BMI) of the subjects.

Methods: Twenty patients were scanned to evaluate DCSA and DLR at three consecutive lumbar spine intervertebral disc levels (L3/4, L4/5, and L5/S1) on conventional-recumbent MRI, and after axial loading were applied.

Results: Axial-loaded MRI demonstrates a significant difference of DSCA in comparison to conventional MRI. Furthermore, results show a significant correlation between the DCSA and BMI on level L3/L4, both before and after axial loading MRI. With axial loading, there is a reduction of DSCA of $12.2 \%, 12.1 \%$, and $2.1 \%$ at the levels L3/L4, L4/L5, and L5/S1, respectively. After axial loading has been applied, the depth of the neural foramen has been reduced by an average of $10.1 \%$.

Conclusion: Axial-loaded MRI reduces DCSA and DLRs in comparison to standard MRI. Information obtained in this way may be useful to explain the patient's symptomatology and may provide an additional insight that can influence the treatment decision plan accordingly.

Keywords: Spinal canal stenosis; axial loading; MRI; lumbar spine; dural sac cross-sectional area; depth lateral recess

\section{INTRODUCTION}

The normal function of the spine is conditioned by its stability. In addition to protecting nerve structures, spinal stability is a basic requirement for transmitting forces between the upper and lower extremities, actively generating torso forces, preventing early biomechanical deterioration of spine components, and reducing energy expenditure during muscle action $(1,2)$.

White et al. defined clinical stability as the ability of the spine to limit movement patterns under physiological loads so as not to damage or irritate the spinal cord and nerve roots, thus preventing disability or pain caused by structural changes (3).

Instability is an important and often unknown cause of back pain, especially at the lumbar level. White and Panjabi

*Corresponding author: Deniz Bulja, Radiology Clinic, Clinical Center University of Sarajevo, Bolnička 25, 71000 Sarajevo, Bosnia and

Herzegovina. E-mail: deniz.bulja@kcus.ba

Submitted: 03 November 2021/Accepted: 13 December 2021 defined spinal instability as the loss of the spine's ability to maintain its movement patterns under physiological load; there is no initial or additional neurological deficit, no significant deformity, or incapacitating pain (4).

Axial loading forces in healthy intervertebral discs are transmitted through the galleon nucleus to vertebral endplates that deflect. The healthy intervertebral disc provides mobility to the spine and transfers load through hydrostatic pressurization of the hydrated nucleus pulposus. Changes to the tissue properties of the disc, including dehydration and reorganization of the nucleus and stiffening of the annulus fibrosus, markedly alter the mechanics of load transfer in the spine that consecutively alters the cancellous bone structure of the affected vertebrae (5).

Although the vertebral body is viewed as a solid non-compressive structure without dynamic potential, vertebral body remodeling due to alterations in the degree of biomechanical stress and/or low-grade vertebral body inflammation in the sense of slight concentric body enlargement, slight vertebral body height reduction, and marginal osteophytosis may lead to occurrence and worsening of spinal 
stenosis when physiological and positional biomechanical changes can provoke neurogenic claudication due to loading of the spine in the upright position (6).

Stenosis due to degenerative changes of the lumbar spine is a condition that can cause compression of neural elements in the vertebral canal (7).

Symptoms of lumbar spinal canal stenosis have certain provocative (neurogenic claudication) and palliative characteristics (i.e., relief of the painful symptoms in anterior flexion, while sitting and/or lying down) (8).

Magnetic resonance imaging (MRI) is considered the method of choice in the diagnosis of degenerative changes of the spine, apart from the detection of the vacuum phenomenon of the disc due to better tissue contrast compared to other diagnostic modalities. It is considered the most useful modality in the evaluation of myelopathy, radiculopathy, and lower back pain. Superior contrast resolution of MRI allows the best view of the soft tissues of the lumbar spine in comparison with standard radiography, myelography, and computed tomography. The spinal cord, nerve roots, cerebrospinal fluid, vertebrae, discs, and ligaments are differentiated and presented in detail.

Ideally, scanning in a standing postural position yields optimal results that truly reflect changes that occur during normal gravitational pressure on the spine. However, this is impractical because the patient would have to stand still for about $30 \mathrm{~min}$ in a specially designed magnetic resonance scanner. To simulate an upright position, clinicians and researchers have developed devices that can axially load the spine in a supine position. These devices typically consist of a non-magnetic harness/jacket with straps connected to the footplate. By tightening or releasing the adjustment buttons on the compression part, the load can be regulated and evenly distributed on both legs. The applied load can be measured using a scale on the footplate (9).

Axial loading MRI provides a level of image detail and insight that is not typically present in conventional MRI because it allows visualization of spinal morphology under axial load. Visualization in these conditions is important since in many instances, low back pain is induced only in an upright or supine position with axial loading due to changes in the form and width of the spinal canal, nerve root compression, intervertebral instability, or disc degeneration, all of which may not be predominant and obvious under reduced load on the back in the supine position $(10,11)$.

In elderly population with degenerative changes of the lumbar spine, the degree of spinal canal stenosis increases. Therefore, some authors believe that older age may be an indication for the use of axial load (12).

\section{METHODS}

Twenty subjects were enrolled in the study, including nine male and 11 female subjects. The age range of the patients was from 27.6 to 68.2 years.

All subjects had low back pain lasting for more than 12 weeks and with varying intensity. Patients with comorbidities, including osteoporosis, traumatic and tumor lesions of the lumbosacral spine, or history of previous surgery in that region, were not enrolled in this study. Patients tolerated the study; no additional pain medications were given. Ethical committee of Clinical center of Sarajevo
University gave positive response no: 06-04-9-19682 for conducing this research. All participants gave written consent for including their data in this research.

The study was performed at the Clinical Center of the University of Sarajevo, at the Clinic of Radiology, using a magnetic resonance device Toshiba Vantage 1.5 Tesla, using a surface coil.

The scanning protocol consisted of two stages of examination.

In the first stage, routine MRI in a recumbent-supine position was performed. After most of the routine MRI has been done, the second stage of the MR examination followed, in which axial loading was applied through a commercially available axial loading device (DynaWell L-Spine; DynaWell Int. AB, Billdal, Sweden) approved by the US Food and Drug Administration.

The device includes a non-magnetic jacket with straps connected to the footplate with a compression mechanism. The jacket can adjust to the patient's posture and is pulled on before the start of routine MRI, and then tightened during the second stage of the examination as axial loading of the sidebands to distribute the pressure to the footrest. The applied load is $50 \%$ of the patient's weight and this load must be equally distributed on both legs, considering the patient's tolerability. The duration of axial loading applied is $10 \mathrm{~min}$ before axial loaded MRI starts. The axial loading is continuously applied with the same intensity during this period.

All subjects in our study were first examined with recumbent MRI in their supine psoas-relaxed position and again after axial loading were applied. In both stages of the examination, sagittal T2-weighted and axial T2-weighted fast spin-echo sequences were obtained. The repetition time/ echo time was 3000/108 for T2-weighted sagittal and $3000 / 108$ for axial images. The slice thickness was $5 \mathrm{~mm}$, and the field of view was $230 / 250 \mathrm{~mm}$ for sagittal and axial images. The imaging matrix was $224 / 320$ for sagittal images and $320 / 320$ for axial images.

The dural sac cross-sectional area (DCSA) and the depth of lateral recess (DLR) at three lumbar spine disc levels (L3/4, L4/5, and L5/S1) were measured for each subject. DCSA, right DLR, and left DLR were measured through a dedicated program on a digital image view station (IMPAX 6.5.2.114 2011, AGFA HealthCare N.V., Mortsel, Belgium).

The depth of lateral recess represents the distance between the superior articular facet and the top part of the pedicle.

DCSA surface and depth of the lateral recess of neural foramina on the right and left sides were determined at the identical intervertebral levels before and after axial loading.

The measured DCSA, right DLR (rDLR), and left DLR (IDLR) values for each specific disc level of all 20 subjects were collected, and mean values with a standard deviation of DCSA, rDLR, and IDLR have been calculated for each of three-disc levels of interest.

The paired Student's t-test was used to determine if a statistically significant difference existed in the mean value at each disc level of DCSA, rDLR, and IDLR between MRI scans before and after axial loading. The percentage decrease in DCSA, rDLR, and IDLR at each level both pre- and post-axial loading was determined. The effect of 
axial loading on DCSA value varied from case to case. In one of the studies on axial loading, a significant decrease in DCSA was defined as a decrease of $\geq 15 \mathrm{~mm}^{2}$ (13).

The results obtained were statistically analyzed in IBM SPSS ver. 26. The mean $\bar{x}$ or median $\mathrm{x}$ was used as measures of central tendency, depending on whether the distribution was normal or not. Furthermore, measures of dispersion were selected based on normality criteria. Hence, standard deviation $(\sigma)$ and interquartile range $(\Delta Q)$ were used, for normal and non-symmetrical distributions, respectively. The normality of the distribution was tested with the Kolmogorov-Smirnov test. In the case of inferential statistics, the Student's t-test and the paired Student's t-test were used for data that follow the normal distribution, while the Mann-Whitney U-test was used for non-symmetrical and parametric data. The Pearson correlation test was used to test the correlation between the variables. The binomial test was used to check the deviation from the expected distribution of variables from two categories.

\section{RESULTS}

The number of male subjects did not significantly differ from the number of female subjects (binomial test, $p=0.824)$, which can also be observed in Table 1 .

The mean age of the subjects was $53(\sigma=10)$, without significant differences between male and female subjects as is shown on the quantile diagram (Graph 1). As expected, gender differences existed in patient body weight and height (Mann-Whitney U-test, $p<0.05$ ). The median body weight and height of male subjects were $90 \mathrm{~kg}$ and $180 \mathrm{~cm}$, respectively, while in women, these values were $80 \mathrm{~kg}$ and $165 \mathrm{~m}$, respectively. On the other hand, the body mass index (BMI) of the patients did not differ significantly between the sexes (Student's t-test, $p=0.564$ ), which can also be observed in the quantile diagram (Graph 2).

The mean BMI was $28.8 \mathrm{~kg} / \mathrm{m}^{2}\left(\sigma=4.5 \mathrm{~kg} / \mathrm{m}^{2}\right)$, which is within the overweight range according to the World Health Organization classification.

Table 2 provides an overview of the mean values and standard deviations of the measured DCSA in patients before and after axial load. The difference between the DCSAs before and after axial loading was significant (paired Student's t-test, $p<0.001)$. After axial loading, there was a reduction of DCSA of $12.2 \%, 12.1 \%$, and $2.1 \%$ at the levels L3/L4, L4/L5, and L5/S1, respectively. There was a significant correlation between the DCSA and BMI on L3/L4, both before and after axial loading have been applied. A significant correlation of DCSA and BMI at the L4/L5 level was present after axial loading.

Table 3 provides an overview of the mean values and standard deviations of the neural foramen depth (left and right) before and after axial loading has been applied. The difference is significant for all sides and conditions (paired Student's t-test, $p<0.05)$. After applying axial loading, the depth of the lateral recess was reduced by an average of $10.1 \%$. At most of the levels examined, there was no significant correlation with BMI, except in the case of the depth of the left recess before axial loading (Pearson correlation test, $p=0.021$ ).

\section{DISCUSSION}

MRI of the lumbosacral spine using axial loading can probably show the actual state of the spinal canal and stenosis if it is present in comparison to routine MRI.

Aggravation of the patient's symptoms in a standing or upright position is due to narrowing of the spinal canal and compression of the nerve roots. Several studies have shown that axial loading affects spinal dynamics and morphological changes during MRI $(14,15)$.

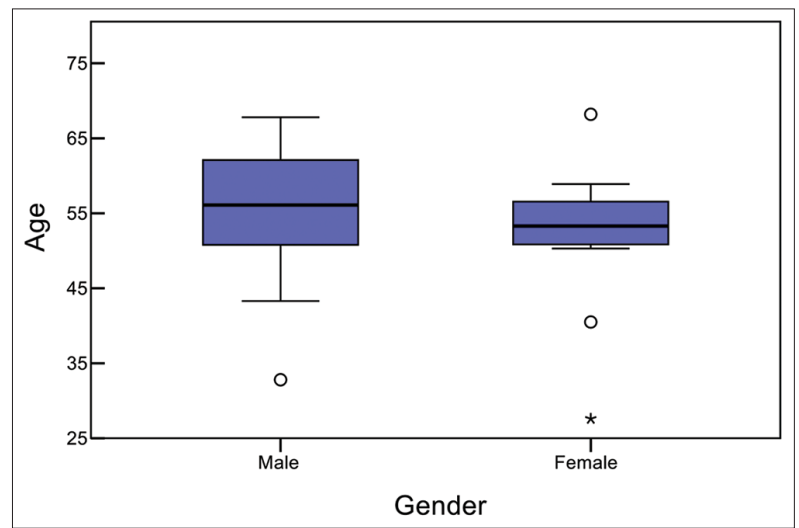

GRAPH 1. Quantile diagram of the age of the subjects enrolled in the study. There are no significant differences between men and women (Student's t-test, $p=0.625$ ).

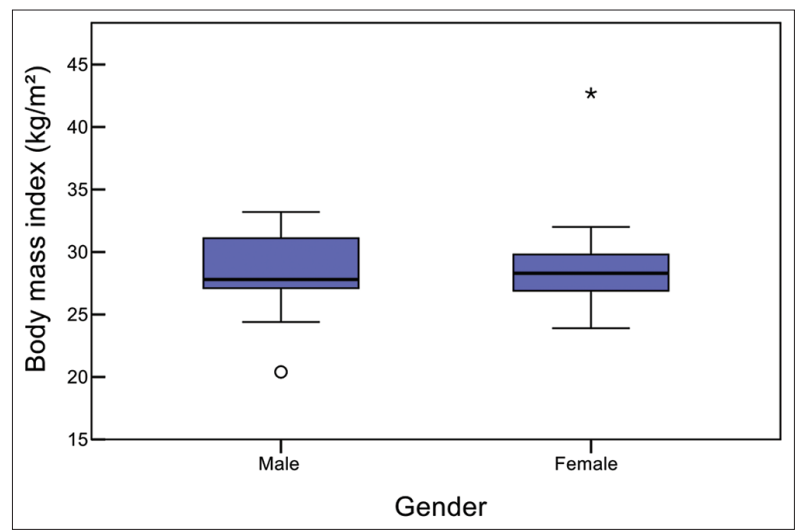

GRAPH 2. Quantile diagram of body mass index of the subjects enrolled in the study. There are no significant differences between men and women (Student's t-test, $p=0.564$ )

TABLE 1. Median $(\tilde{x})$ and interquartile range $(\Delta Q)$ of age, body weight, and height of patients included in the study, grouped by gender and total

\begin{tabular}{|c|c|c|c|c|c|c|}
\hline & \multicolumn{2}{|c|}{ Gender } & & & \multicolumn{2}{|c|}{ Total $(N=20)$} \\
\hline & \multicolumn{2}{|c|}{ Male $(N=9)$} & \multicolumn{2}{|c|}{ Female $(N=11)$} & & \\
\hline & $\tilde{\mathrm{X}}$ (min-max) & $\Delta Q$ & $\tilde{\mathrm{X}}$ (min-max) & $\Delta \mathrm{Q}$ & $\tilde{X}(\min -\max )$ & $\Delta Q$ \\
\hline Age range $^{\mathrm{a}}$ & $56.1(32.8-67.8)$ & 11.3 & $53.3(27.6-68.2)$ & 7.2 & $53.8(27.6-68.2)$ & 7.9 \\
\hline Body mass $(\mathrm{kg})^{\mathrm{b}}$ & 90 & 11 & 80 & 10 & 83 & 11 \\
\hline Body height $(m)^{b}$ & 180 & 7 & 165 & 6 & 170 & 14 \\
\hline
\end{tabular}

aDistribution does not follow the normal distribution (Kolmogorov-Smirnov test, $P=0.028$ ), bthe difference between the sexes is significant (Mann-Whitney U-test, $P<0.05)$ 
TABLE 2. Dural sac area before and after compression (mean $\tilde{X}$ and standard deviation $\sigma$ ) depending on BMI and total

\begin{tabular}{|c|c|c|c|c|c|c|c|c|c|}
\hline \multirow[t]{3}{*}{ Axial loading } & \multicolumn{6}{|c|}{$\mathrm{BMI}\left(\mathrm{kg} / \mathrm{m}^{2}\right)$} & \multirow{2}{*}{\multicolumn{2}{|c|}{ Total }} & \multirow[t]{3}{*}{$p$} \\
\hline & \multicolumn{2}{|c|}{$18.5-24.9$} & \multicolumn{2}{|c|}{$25-29.9$} & \multicolumn{2}{|c|}{$>30$} & & & \\
\hline & $\tilde{X}$ & $\sigma$ & $\tilde{X}$ & $\sigma$ & $\tilde{X}$ & $\sigma$ & $\tilde{X}$ & $\sigma$ & \\
\hline \multicolumn{10}{|c|}{$\mathrm{DCSA}(\mathrm{L} 3 / \mathrm{L} 4)\left(\mathrm{mm}^{2}\right)$} \\
\hline Before $^{a}$ & 181.4 & 15.7 & 146.3 & 45.8 & 105.3 & 32.3 & 139.3 & 45.6 & $<0.001$ \\
\hline After ${ }^{\mathrm{a}}$ & 167.0 & 9.5 & 131.3 & 42.6 & 83.4 & 36.6 & 122.3 & 46.5 & \\
\hline \multicolumn{10}{|c|}{ DCSA (L4/L5) (mm²) } \\
\hline Before & 150.5 & 19.2 & 142.3 & 50.5 & 96.4 & 34.1 & 129.7 & 46.9 & $<0.001$ \\
\hline Aftera & 138.8 & 12.6 & 128.7 & 48.8 & 74.8 & 37.8 & 114.0 & 48.5 & \\
\hline \multicolumn{10}{|c|}{$\mathrm{DCSA}(\mathrm{L} 5 / \mathrm{S} 1)\left(\mathrm{mm}^{2}\right)$} \\
\hline Before & 151.7 & 39.6 & 179.2 & 48.1 & 119.1 & 58.0 & 157.1 & 54.9 & $<0.001$ \\
\hline After & 138.0 & 37.3 & 176.5 & 41.1 & 120.0 & 66.6 & 153.8 & 53.9 & \\
\hline
\end{tabular}

There was a significant correlation between the DCSA and body mass index (Pearson correlation test, $P<0.05$ ).DCSA: Dural sac cross-sectional area, BMl: Body mass index

TABLE 3. DLR, right and left, before and after axial loading (mean X̃and standard deviation $\sigma$ ) depending on BMI and total

\begin{tabular}{|c|c|c|c|c|c|c|c|c|c|c|}
\hline & \multirow[t]{3}{*}{ Axial loading } & \multicolumn{8}{|c|}{$\mathrm{BMI}\left(\mathrm{kg} / \mathrm{m}^{2}\right)$} & \multirow[t]{3}{*}{$p$} \\
\hline & & \multicolumn{2}{|c|}{$18.5-24.9$} & \multicolumn{2}{|c|}{$25-29.9$} & \multicolumn{2}{|c|}{$>30$} & \multicolumn{2}{|c|}{ Total } & \\
\hline & & $\tilde{X}$ & $\sigma$ & $\tilde{x}$ & $\sigma$ & $\tilde{X}$ & $\sigma$ & $\tilde{X}$ & $\sigma$ & \\
\hline \multirow[t]{2}{*}{ rDLR (L3/L4) (mm) } & Before & 4.5 & 1.5 & 5.3 & 2.6 & 6.0 & 2.2 & 5.4 & 2.3 & $<0.001$ \\
\hline & After & 4.1 & 1.5 & 4.7 & 2.5 & 5.6 & 2.1 & 4.9 & 2.2 & \\
\hline \multirow[t]{2}{*}{ IDLR (L3/L4) (mm) } & Before & 3.3 & 1.0 & 4.8 & 2.3 & 6.4 & 2.8 & 5.0 & 2.4 & 0.001 \\
\hline & After & 3.0 & 1.1 & 4.3 & 2.2 & 5.2 & 2.1 & 4.4 & 2.1 & \\
\hline \multirow[t]{2}{*}{ rDLR (L4/L5) (mm) } & Before & 4.3 & 0.8 & 4.5 & 1.8 & 6.5 & 1.5 & 5.1 & 1.8 & 0.003 \\
\hline & After & 4.1 & 0.9 & 4.2 & 1.6 & 5.7 & 1.0 & 4.6 & 1.5 & \\
\hline \multirow[t]{2}{*}{ IDLR (L4/L5) (mm) } & Before $^{a}$ & 4.2 & 1.6 & 4.3 & 2.0 & 7.8 & 2.9 & 5.3 & 2.7 & 0.012 \\
\hline & After & 3.9 & 1.9 & 4.1 & 1.9 & 6.2 & 1.5 & 4.7 & 2.0 & \\
\hline \multirow{2}{*}{$\begin{array}{l}\text { rDLR (L5/S1) } \\
(\mathrm{mm})\end{array}$} & Before & 6.5 & 2.8 & 6.1 & 2.2 & 5.1 & 2.0 & 5.8 & 2.2 & 0.001 \\
\hline & After & 5.8 & 2.6 & 5.7 & 2.1 & 4.3 & 1.7 & 5.3 & 2.1 & \\
\hline \multirow[t]{2}{*}{ IDLR (L5/S1) (mm) } & Before & 5.8 & 3.5 & 4.8 & 1.6 & 5.5 & 1.8 & 5.2 & 1.9 & 0.013 \\
\hline & After & 5.6 & 3.5 & 4.6 & 1.7 & 4.5 & 1.0 & 4.7 & 1.8 & \\
\hline
\end{tabular}

aThere is a significant correlation between neural foramen depth and body mass index (Pearson correlation test, $P=0.021$ ). DLR: Depth of lateral recess, rDLR: Right depth of lateral recess, IDLR: Left depth of lateral recess, BMI: Body mass index

The rationale for this study was to determine whether adding an axially loaded segment of MRI of the lumbar spine could yield information that would suggest or be in concordance with patient symptomatology, thus influencing or altering final treatment decision.

To show the effects of axial loading, we determined that the DCSA at the levels of intervertebral discs L3/L4, L4/L5, and L5/S1 before and after axial loading has been applied. The percentage of the DCSA reduction in axial loaded scans varied and was probably related to differences in body resistance to load. Further study is needed to define the correlation between spinal stenosis and body resistance (16).

Axial loading causes a reduction in the area and diameter of the dural sac at L3/L4 and L4/L5, and, to a lesser extent, at the level of the lumbar spine than L5/S1, which is probably due to paradoxical motion exerted at this level (17).

Willén and Danielson study of the effect of axial loading on the lumbar spine in suspected lumbar spinal stenosis reported that the axial load significantly reduced the cross-sectional area of the dural sac $(18,19)$.

Herniated discs increased in size in the axial plane leading to an augmentation in nerve root compression grades for paracentral herniated discs in the standing position compared with those in the conventional supine position. Weight-bearing MRI may increase the diagnostic sensitivity of disc herniations in patients suspected of nerve root compression (20).
Guideline of the North American Spine Society suggests axial loading for when there is symptomatic spinal canal stenosis in the lumbosacral spine (21).

There was a significant difference in DCSA in axial loading MRI in $76 \%$ of patients with suspected spinal stenosis (22).

Splendiani et al. claimed that in the pathogenesis of radiculopathy in some patients lies dynamic stenosis of the neural foramen is caused by the physiological axial load (23).

A method of estimating the width of the spinal canal through the calculation of the cross-sectional area of the dural sac at the level of the intervertebral spaces of the lumbar spine has been proposed by Hamanishi et al. (13).

The widely accepted radiological threshold for early stenosis is the area of the dural sac between 100 and $130 \mathrm{~mm}^{2}$. According to these authors, the relative stenosis of the spinal canal at the level of the lumbar spine can be declared at the surface of the dural sac between 75 and $100 \mathrm{~mm}^{2}$, and absolute stenosis exists when the surface of the dural sac is $<75 \mathrm{~mm}^{2}$ in cross section (24).

To the best of our knowledge that is based on PubMed research, there are only three articles that mention a relation of BMI of the subjects who underwent axial loading MRI of the lumbar spine. We did not encounter a study that investigated the possible relation between excessive BMI and the extent of change in lateral recess depth. 
A disadvantage of this study is the number of patients included in the research and the lack of other quantitative assessments such as the surface of the lateral recesses or the angles which could provide further, in-depth insight. One of the disadvantages of the axial loading technique is that it takes a longer time than the usual MRI, by 5-10 min, which can be challenging in terms of cost-effectiveness, thus, it may provoke pain during the procedure (25).

Results of this study suggest that axial-loaded MRI may provide additional and perhaps more accurate information for the clinical assessment of patients with symptoms associated with lumbar neurological compromise.

MRI with axial loading could be appropriate for patients with excessive BMI who have symptoms associated with lumbar neurological compromise and in whom routine MRI cannot explain clinical symptoms. Increased spinal canal stenosis after axial loading can change the treatment plan for the patient.

It is suggested to use axial loading if there is no medical contraindication, such as osteoporosis, traumatic, or tumor lesions of the lumbosacral spine, especially in patients whose routine MRI results do not match their clinical symptoms.

\section{CONCLUSION}

MRI with axial loading can provide more information on vertebral alignment, intervertebral disc height, spinal canal width, and ligamentum flavum hypertrophy; this information can explain the patient's symptomatology and can significantly change the treatment decision plan accordingly.

Axial loading reduces DCSA; it, therefore, increases the severity of lumbar canal stenosis, and the effect of axial loading on MRI examination is greatest at L3/L4, L4/L5, and, to a lesser extent, at L5/S1 levels, probably due to paradoxical motion at this level.

Patients with clinical symptoms that could not be explained by conventional-recumbent MRI may benefit from axial load MRI which can be beneficial in providing additional information that may affect the future treatment decision plan.

\section{DECLARATION OF INTERESTS}

Authors declare no conflict of interest.

\section{REFERENCES}

1. Haher TR, O' Brien M, Kauffman D, Liao KC. Biomechanics of the spine in sports. Clin Sports Med 1993;12(3):449-64

2. Guillot M, Fournier J, Vanneuville G, Galtier B, Tanguy A, Scheye T, et al. Methods of measuring the mechanical stresses on the human lumbar spine and their results. Rev Rhum Mal Osteoartic 1988;55(5):351-9.

3. White AA, Johnson RM, Panjabi MM, Southwick WO. Biomechanical analysis of clinical stability in the cervical spine. Clin Orthop Relat Res 1975;109:85-96. https://doi.org/10.1097/00003086-197506000-00011

4. White AA, Panjabi MM. The basic kinematics of the human spine. Spine (Phila Pa 1976) 1978;3(1):12-20.

https://doi.org/10.1097/00007632-197803000-00003

5. Ferguson SJ, Steffen T. Biomechanics of the aging spine. Eur Spine J 2003;12 Suppl 2:S97-S103.

https://doi.org/10.1007/s00586-003-0621-0
6. Hansen BB, Nordberg CL, Hansen P, Bliddal H, Griffith JF, Fournier G, et al. Weightbearing MRI of the lumbar spine: Spinal stenosis and spondylolisthesis. Semin Musculoskelet Radiol 2019;23(6):621-33 https://doi.org/10.1055/s-0039-1697937

7. Covaro A, Vilà-Canet G, de Frutos AG, Ubierna MT, Ciccolo F, Caceres E. Management of degenerative lumbar spinal stenosis: An evidence-based review. EFORT Open Rev 2017;1(7):267-74

\section{https://doi.org/10.1302/2058-5241.1.000030}

8. Otani K, Kikuchi SI, Nikaido T, Konno SI. Magnitude of dural tube compression does not show a predictive value for symptomatic lumbar spinal stenosis for 1-year follow-up: A prospective cohort study in the community. Clin Interv Aging 2018;13:1739-46. https://doi.org/10.2147/CIA.S171049

9. Hiwatashi A, Danielson B, Moritani T, Bakos RS, Rodenhause TG, Pilcher WH, et al. Axial loading during MR imaging can influence treatment decision for symptomatic spinal stenosis. AJNR Am J Neuroradiol 2004;25(2):170-4.

10. Haynes S, Williams K. Impact of seating posture on user comfort and typing performance for people with chronic low back pain. Int J Ind Ergon 2008;38(1):35-46. https://doi.org/10.1016/j.ergon.2007.08.003

11. Weishaupt $D$, Boxheimer $L$. Magnetic resonance imaging of the weight-bearing spine. Semin Musculoskelet Radiol 2003;7(4):277-86

https://doi.org/10.1055/s-2004-815675

12. Sasani H, Solmaz B, Sasani M, Vural M, Ozer AF. Diagnostic importance of axial loaded magnetic resonance imaging in patients with suspected lumbar spinal canal stenosis. World Neurosurg 2019;127:e69-75.

https://doi.org/10.1016/j.wneu.2019.02.091

13. Hamanishi C, Matukura N, Fujita M, Tomihara M, Tanaka S. Cross-sectional area of the stenotic lumbar dural tube measured from the transverse views of magnetic resonance imaging. J Spinal Disord 1994;7(5):388-93.

14. Meakin JR, Smith FW, Gilbert FJ, Aspden RM. The effect of axial load on the sagittal plane curvature of the upright human spine in vivo. J Biomech 2008;41(13):2850-4.

https://doi.org/10.1016/j.jbiomech.2008.06.035

15. O'Connell GD, Johannessen W, Vresilovic EJ, Elliott DM. Human internal disc strains in axial compression measured noninvasively using magnetic resonance imaging. Spine (Phila Pa 1976) 2007;32(25):2860-8.

https://doi.org/10.1097/BRS.0b013e31815b75fb

16. Wang YC, Jeng CM, Wu CY, Chang HY, Huang YC, Wang YJ, et al. Dynamic effects of axial loading on the lumbar spine during magnetic resonance imaging in patients with suspected spinal stenosis. J Formos Med Assoc 2008;107(4):334-9.

https://doi.org/10.1016/S0929-6646(08)60095-9

17. Oh JY, Liang S, Louange D, Rahmat R, Hee HT, Kumar VP. Paradoxical motion in L5-S1 adult spondylolytic spondylolisthesis. Eur Spine J 2012;21(2):262-7. https://doi.org/10.1007/s00586-011-1880-9

18. Willén J, Danielson B. The diagnostic effect from axial loading of the lumbar spine during computed tomography and magnetic resonance imaging in patients with degenerative disorders. Spine (Phila Pa 1976) 2001;26:2607-14.

19. Hansson T, Suzuki N, Hebelka H, Gaulitz A. The narrowing of the lumbar spinal canal during loaded MRI: The effects of the disc and ligamentum flavum. Eur Spine J 2009;18(5):679-86. https://doi.org/10.1007/s00586-009-0919-7

20. Nordberg CL, Boesen M, Fournier GL, Bliddal H, Hansen P, Hansen BB. Positional changes in lumbar disc herniation during standing or lumbar extension: A cross-sectional weight-bearing MRI study. Eur Radiol 2021;31(2):804-12. https://doi.org/10.1007/s00330-020-07132-w

21. North American Spine Society. Diagnosis and Treatment of Degenerative Lumbar Spinal Stenosis. Burr Ridge, Illinois: North American Spine Society; 2019. Available from: https://www.spine.org/documents/researchclinicalcare/guidelines/lumbarstenosis.pdf [Last accessed on 2019 Oct 20].

22. Danielson BI, Willén J, Gaulitz A, Niklason T, Hansson TH. Axial loading of the spine during CT and MR in patients with suspected lumbar spinal stenosis. Acta Radiol 1998;39(6):604-11. https://doi.org/10.3109/02841859809175484

23. Splendiani A, Ferrari F, Barile A, Masciocchi C, Gallucci M. Occult neural foraminal stenosis caused by association between disc degeneration and facet joint osteoarthritis: Demonstration with dedicated upright MRI system. Radiol Med 2014;119(3):164-74. https://doi.org/10.1007/s11547-013-0330-7

24. Schonstrom N, Lindahl S, Willen J, Hansson T. Dynamic changes in the dimensions of the lumbar spinal canal: An experimental study in vitro. J Orthop Res 1989;7(1):115-21. https://doi.org/10.1002/jor.1100070116

25. Ali HI, Saleh A. Lumbar spine MRI axial loading in patients with degenerative spine pathologies: Its impact on the radiological findings and treatment decision. Egypt $J$ Radiol Nucl Med 2015;46(4):1065-9.

\section{RELATED ARTICLES PUBLISHED IN JHSCI}

1. Hasanović-Vučković S, Jusufbegović M, Vegar-Zubović S, Milišić L, Šehić A, Hasanbegović I, Beganović A. Assessment of Lumbar Spine Disc Degeneration in Coherence to Pfirrman Grades and Oswestry Disability Index. JHSCI. 2020;10(3):191-5. 